PROCEEDINGS OF THE

AMERICAN MATHEMATICAL SOCIETY

Volume 127, Number 11, Pages 3403-3412

S 0002-9939(99)04948-5

Article electronically published on May 3, 1999

\title{
MATRIX PRESENTATIONS OF BRAIDS AND APPLICATIONS
}

\author{
SANG YOUL LEE
}

(Communicated by Ronald A. Fintushel)

\begin{abstract}
We show that there exists a one-to-one correspondence between the class of certain block tridiagonal matrices with the entries $-1,0$, or 1 and the free monoid generated by $2 n$ generators $\sigma_{1}, \cdots, \sigma_{n}, \sigma_{1}^{-1}, \cdots, \sigma_{n}^{-1}$ and relation $\sigma_{i}^{ \pm 1} \sigma_{j}^{ \pm 1}=\sigma_{j}^{ \pm 1} \sigma_{i}^{ \pm 1}(|i-j| \geq 2)$ and give some applications for braids. In particular, we give new formulation of the reduced Alexander matrices for closed braids.
\end{abstract}

\section{INTRODUCTION}

The Artin's braid group on $n+1$ strings $B_{n+1}(n>0)$ has a standard presentation as a group with generators $\sigma_{1}, \sigma_{2}, \cdots, \sigma_{n}$ and defining relations:

$$
\begin{aligned}
& \text { (I) } \sigma_{i} \sigma_{j}=\sigma_{j} \sigma_{i}(|i-j| \geq 2), \\
& \text { (II) } \sigma_{i} \sigma_{i+1} \sigma_{i}=\sigma_{i+1} \sigma_{i} \sigma_{i+1}(1 \leq i \leq n-1) .
\end{aligned}
$$

By $(\gamma, n+1)$ we mean a braid $\gamma$ in $B_{n+1}$. The closure of a braid $(\gamma, n+1)$ is denoted by $(\gamma, n+1)^{\wedge}$ or simply by $\gamma^{\wedge}$. In [Al], Alexander showed that every oriented link in $S^{3}$ is ambient isotopic to the closure of some braid and Markov's theorem [Bir] said that two braids have the ambient isotopic closures if and only if they are Markov equivalent, i.e., there exists a finite sequence of the following two moves taking one to the other: $(M 1)$ Replace $(\gamma, n+1)$ by $(\omega, n+1)$, where $\omega$ is a conjugate of $\gamma$; (M2) Replace $(\gamma, n+1)$ by $\left(\gamma \sigma_{n+1}^{ \pm 1}, n+2\right)$ (or vice versa).

In section 2 of this paper, we show that the class $\Omega_{n}$ of certain block tridiagonal matrices with entries $-1,0$, or 1 and the free monoid $\mathcal{F}_{n}$, under juxtaposition, of all words generated by $2 n$ generators $\sigma_{1}, \cdots, \sigma_{n}, \sigma_{1}^{-1}, \cdots, \sigma_{n}^{-1}$ are in one-to-one correspondence up to the relation $\sigma_{i}^{ \pm 1} \sigma_{j}^{ \pm 1}=\sigma_{j}^{ \pm 1} \sigma_{i}^{ \pm 1}(|i-j| \geq 2)$ in $\mathcal{F}_{n}$. Since a braid word $\gamma$ in $B_{n+1}$ can be regarded as an element of $\mathcal{F}_{n}$, this correspondence gives a matrix presentation $M(\gamma) \in \Omega_{n}$ of the braid $\gamma$ and, conversely, the matrix $M(\gamma)$ reproduces the braid $\gamma$ up to the defining relation (I).

In [Mur], Murasugi defined an integral matrix $M$, called Murasugi's matrix, with respect to an oriented link diagram $L$ in $S^{2}$ and showed that the $S$-equivalence class of the symmetrized matrix $M^{*}+M^{* T}$ of its principal minor $M^{*}$ is an oriented link type invariant. He thereby attached a class of quadratic forms to an oriented link $L$ and defined the signature of $L$, denoted by $\sigma(L)$, to be the signature of $M^{*}+M^{* T}$

Received by the editors July 31, 1997 and, in revised form, January 26, 1998.

1991 Mathematics Subject Classification. Primary 57M25; Secondary 20 F36.

Key words and phrases. Alexander matrix, braid, nullity, signature.

This research was supported by the Korea Science and Engineering Foundation.

(C)1999 American Mathematical Society 
and the nullity of $L$, denoted by $\mathcal{N}(L)$, to be the nullity of $M^{*}+M^{* T}$ plus 1 . Some authors ([GL], [Tr]) developed more simple algorithms to compute the signature and nullity of an oriented link via its link diagram.

In section 3, for a braid $\gamma \in B_{n+1}$, we characterize a Murasugi's matrix with respect to the closure $\gamma^{\wedge}$ of $\gamma$ from the matrix presentation $M(\gamma) \in \Omega_{n}$ of $\gamma$ by using simple matrix calculations. This gives a new formulation of a reduced Alexander matrix $\Lambda_{M(\gamma)}(t)$ for the closure of a braid $\gamma$ and simultaneously gives an algorithm to compute the signature and nullity of the closure $\gamma^{\wedge}$ of $\gamma$ by using the reduced Alexander matrix $\Lambda_{M(\gamma)}(t)$ for $\gamma^{\wedge}$ without using the braid version of Reidemeister move III.

\section{Certain Block Matrices AND WORds}

Let $A$ be a block tridiagonal matrix of the form

$$
A=\left(\begin{array}{cccccc}
A_{11} & A_{12} & & & & \\
A_{21} & A_{22} & A_{23} & & & \\
& A_{32} & A_{33} & A_{34} & & \\
& & \ddots & \ddots & \ddots & \\
& & & A_{n-1 n-2} & A_{n-1 n-1} & A_{n-1 n} \\
& & & & A_{n n-1} & A_{n n}
\end{array}\right)
$$

satisfying the following conditions: for each $i \in\{1,2, \cdots, n\}$,

(2.1.1). Each diagonal block $A_{i i}=(0)$, the $1 \times 1$ zero matrix, or the $s_{i} \times s_{i}$ matrix of the form: for $\tau_{k}= \pm 1\left(k=1,2, \cdots, s_{i}\right), A_{i i}=\left(-\tau_{1}\right)\left(s_{i}=1\right)$, and

$$
A_{i i}=\left(\begin{array}{cccccc}
-\tau_{1} & 0 & 0 & \cdots & 0 & \tau_{1} \\
\tau_{2} & -\tau_{2} & 0 & \cdots & 0 & 0 \\
0 & \tau_{3} & -\tau_{3} & \cdots & 0 & 0 \\
\vdots & \vdots & \vdots & \ddots & \vdots & \vdots \\
0 & 0 & 0 & \cdots & -\tau_{s_{i}-1} & 0 \\
0 & 0 & 0 & \cdots & \tau_{s_{i}} & -\tau_{s_{i}}
\end{array}\right)\left(s_{i}>1\right) .
$$

(2.1.2). If $A_{i i}=(0)$, then $A_{i i-1}, A_{i i+1}, A_{i-1 i}$, and $A_{i+1 i}$ are all zero matrices.

If $A_{i i}, A_{i+1 i+1} \neq(0)$, let $c_{j}\left(j=1,2, \cdots, s_{i}\right)$ denote the $j^{\text {th }}$-column vector of the matrix $A_{i+1 i}$, i.e., $A_{i+1 i}=\left[\begin{array}{lllll}c_{1} & c_{2} & \cdots & c_{s_{i}}\end{array}\right]$. Then $A_{i+1 i}$ and $A_{i i+1}$ satisfies the following:

(1) One and only one of the following occurs.

(i) All $c_{j}\left(j=1,2, \cdots, s_{i}\right)$ are nonzero and $A_{i i+1}=O_{s_{i} \times s_{i+1}}$.

(ii) For some $k\left(1 \leq k \leq s_{i}\right), c_{1}, \cdots, c_{k}$ are all zero and $c_{k+1}, \cdots, c_{s_{i}}$ are all nonzero and, in this case, $A_{i i+1}=\left[\begin{array}{lllll}r_{1} & \cdots & r_{k} O & \cdots & O\end{array}\right]^{T}$, where $r_{j}=\left(\begin{array}{llll}0 & \cdots & 0 & 1\end{array}\right)$ or $(0 \cdots 0-1)$ for all $j=1,2, \cdots, k$.

(2) Each nonzero column (resp., row) of $A_{i+1 i}$ (resp., $A_{i i+1}$ ) contains one and only one nonzero entry which is equal to -1 times the diagonal entry of $A_{i i}$ corresponding to the column(resp., row).

(3) Let $c_{k}$ be the first nonzero column of $A_{i+1 i}$. Then for each $p\left(k \leq p \leq s_{i}-1\right)$, the nonzero entry of the $(p+1)^{t h}$-column $c_{p+1}$ of $A_{i+1 i}$ cannot occur in the higher row than the row in which the nonzero entry of the $p^{t h}$-column $c_{p}$ of $A_{i+1 i}$ occurs. 
Let $\Omega_{n}$ denote the set of all block tridiagonal matrices described in (2.1) and let $\mathcal{F}_{n}$ be the free monoid, under juxtaposition, of all words generated by $2 n$ generators $\sigma_{1}, \sigma_{2}, \cdots, \sigma_{n}, \sigma_{1}^{-1}, \sigma_{2}^{-1}, \cdots, \sigma_{n}^{-1}(n \geq 1)$.

For a matrix $A=\left(A_{i j}\right)_{1 \leq i, j \leq n} \in \Omega_{n}$, we define a word $W(A) \in \mathcal{F}_{n}$ by the following procedure:

First, let

$$
W_{n}=\sigma_{n}^{\tau_{1}} \sigma_{n}^{\tau_{2}} \cdots \sigma_{n}^{\tau_{s_{n}}}
$$

where $-\tau_{j}\left(j=1, \cdots, s_{n}\right)$ is the $(j, j)$-diagonal entry of the block $A_{n n}$ of $A$. If $A_{n n}=(0)$, then $W_{n}$ is the empty word.

Next, for $i \in\{1,2, \cdots, n-1\}$, we define the word $W_{i}$ from the word $W_{i+1}$ as follows: If $A_{i i}=(0)$, then $W_{i}=W_{i+1}$.

Now we assume that $A_{i i} \neq(0)$. If $A_{i+1 i+1}=(0)$, then

$$
W_{i}=\sigma_{i}^{\tau_{1}} \sigma_{i}^{\tau_{2}} \cdots \sigma_{i}^{\tau_{s_{i}}} W_{i+1}
$$

If $A_{i+1 i+1} \neq(0)$, then we define $W_{i}$ to be the word obtained from $W_{i+1}$ by inserting the letters $\sigma_{i}^{\tau_{1}}, \sigma_{i}^{\tau_{2}}, \cdots, \sigma_{i}^{\tau_{s_{i}}}$ into the word $W_{i+1}$, where $-\tau_{j}\left(j=1, \cdots, s_{i}\right)$ is the $(j, j)$-diagonal entry of the block $A_{i i}$ of $A$, as follows:

(1) If $A_{i+1 i}=O_{s_{i+1} \times s_{i}}$, then

$$
W_{i}=\sigma_{i}^{\tau_{1}} \sigma_{i}^{\tau_{2}} \cdots \sigma_{i}^{\tau_{s_{i}}} W_{i+1}
$$

(2) If $A_{i+1 i} \neq O_{s_{i+1} \times s_{i}}$ and the $k^{t h}$-column is the first nonzero column of $A_{i+1 i}$, then for each $q=k, \cdots, s_{i}$,

(i) If the nonzero entry of the $q^{t h}$-column occurs in the $p^{t h}$-row $(1 \leq p \leq$ $\left.s_{i+1}\right)$ and the nonzero entry of the $(q-1)^{t h}$-column does not occur in the same $p^{t h}$-row, then insert $\sigma_{i}^{\tau_{1}} \sigma_{i}^{\tau_{2}} \cdots \sigma_{i}^{\tau_{k-1}}$ before $\sigma_{i+1}^{\tau_{1}}$ and insert $\sigma_{i}^{\tau_{q}}$ after $\sigma_{i+1}^{\tau_{p}}$, i.e.,

$$
W_{i}=\cdots \sigma_{i}^{\tau_{1}} \sigma_{i}^{\tau_{2}} \cdots \sigma_{i}^{\tau_{k-1}} \sigma_{i+1}^{\tau_{1}} \cdots \sigma_{i+1}^{\tau_{p}} \sigma_{i}^{\tau_{q}} \cdots \sigma_{i+1}^{\tau_{p+1}} \cdots
$$

(ii) If the nonzero entry of the $q^{\text {th }}$-column $(q \geq k+1)$ occurs in the $p^{\text {th }}$-row $\left(1 \leq p \leq s_{i+1}\right)$ and the nonzero entry of the $(q-1)^{t h}$-column also occurs in the same $p^{t h}$-row, then insert $\sigma_{i}^{\tau_{1}} \sigma_{i}^{\tau_{2}} \cdots \sigma_{i}^{\tau_{k-1}}$ before $\sigma_{i+1}^{\tau_{1}}$ and insert $\sigma_{i}^{\tau_{q}}$ after $\sigma_{i}^{\tau_{q-1}}$, i.e.,

$$
W_{i}=\cdots \sigma_{i}^{\tau_{1}} \sigma_{i}^{\tau_{2}} \cdots \sigma_{i}^{\tau_{k-1}} \sigma_{i+1}^{\tau_{1}} \cdots \sigma_{i+1}^{\tau_{p}} \cdots \sigma_{i}^{\tau_{q-1}} \sigma_{i}^{\tau_{q}} \cdots \sigma_{i+1}^{\tau_{p+1}} \cdots
$$

Repeating this process for $i=n-1, n-2, \cdots, 2,1$, we obtain the word $W_{1} \in \mathcal{F}_{n}$. Finally, we define the word $W(A)$ to be the word $W_{1}$.

Now we are going to consider the reverse procedure. For a word $\gamma=\sigma_{i_{1}}^{\tau_{i_{1}}} \sigma_{i_{2}}^{\tau_{i_{2}}} \ldots$ $\sigma_{i_{m}}^{\tau_{i_{m}}}\left(\tau_{i_{k}}= \pm 1, k=1,2, \cdots, m\right) \in \mathcal{F}_{n}$, we define a block matrix $M(\gamma)=\left(A_{i j}\right)_{1 \leq i, j \leq n}$ $\in \Omega_{n}$ as follows:

For each $i \in\{1,2, \cdots, n\}$, let $s_{i}\left(s_{i} \geq 0\right)$ denote the number of the generators $\sigma_{i}, \sigma_{i}^{-1}$ which occur in the word $\gamma$ and rewrite the $s_{i}$ generators as $\sigma_{(i, 1)}^{\tau(i, 1)}, \sigma_{(i, 2)}^{\tau(i, 2)}, \cdots$, $\sigma_{\left(i, s_{i}\right)}^{\tau\left(i, s_{i}\right)}$ keeping the order from left to right, where $\tau(i, k)$ denotes the exponent of the generator $\sigma_{i}$ in $\gamma$ which constitutes $\sigma_{(i, k)}$ and $s_{1}+s_{2}+\cdots+s_{n}=m$. The resultant is denoted by $\bar{\gamma}$ and called the rewriting word of $\gamma$ (see Example 2.1). 
Each diagonal block $A_{i i}(1 \leq i \leq n)$ of $M(\gamma)$ is defined to be the square matrix given by $A_{i i}=(0)\left(s_{i}=0\right), A_{i i}=(-\tau(i, 1))\left(s_{i}=1\right)$, and

$$
A_{i i}=\left(\begin{array}{cccccc}
-\tau(i, 1) & 0 & 0 & \cdots & 0 & \tau(i, 1) \\
\tau(i, 2) & -\tau(i, 2) & 0 & \cdots & 0 & 0 \\
0 & \tau(i, 3) & -\tau(i, 3) & \cdots & 0 & 0 \\
\vdots & \vdots & \vdots & \ddots & \vdots & \vdots \\
0 & 0 & 0 & \cdots & -\tau\left(i, s_{i}-1\right) & 0 \\
0 & 0 & 0 & \cdots & \tau\left(i, s_{i}\right) & -\tau\left(i, s_{i}\right)
\end{array}\right)\left(s_{i}>1\right)
$$

For $i \neq j$, define $A_{i j}$ as follows:

(1) For $|i-j|>1, A_{i j}=O_{s_{i} \times s_{j}}$.

(2) For $|i-j|=1$, if $s_{i}=0$ and so $A_{i i}=(0)$, then $A_{i i-1}=O_{1 \times s_{i-1}}, A_{i i+1}=$ $O_{1 \times s_{i+1}}, A_{i-1 i}=O_{s_{i-1} \times 1}$, and $A_{i+1 i}=O_{s_{i+1} \times 1}$. If $s_{i} \geq 1$, then

$$
A_{i+1 i}=\left(a_{p q}^{i+1 i}\right)_{1 \leq p \leq s_{i+1}, 1 \leq q \leq s_{i}},
$$

where $a_{p q}^{i+1 i}=\tau(i, q)$ if $1 \leq p \leq s_{i+1}-1$ and $\sigma_{(i, q)}^{\tau(i, q)}$ occurs between $\sigma_{(i+1, p)}^{\tau(i+1, p)}$ and $\sigma_{(i+1, p+1)}^{\tau(i+1, p+1)}$ in $\bar{\gamma}, a_{s_{i+1}}^{i+1 i}=\tau(i, q)$ if $\sigma_{(i, q)}^{\tau(i, q)}$ occurs farther to the right than $\sigma_{\left(i+1, s_{i+1}\right)}^{\tau\left(i+1, s_{i+1}\right)}$ in $\bar{\gamma}$; otherwise, $a_{p q}^{i+1 i}=0$, and

$$
A_{i i+1}=\left(a_{p q}^{i i+1}\right)_{1 \leq p \leq s_{i}, 1 \leq q \leq s_{i+1}},
$$

where $a_{p q}^{i i+1}=\tau(i, p)$ if $q=s_{i+1}$ and $\sigma_{(i, p)}^{\tau(i, p)}$ occurs farther to the left than $\sigma_{(i+1,1)}^{\tau(i+1,1)}$ in $\bar{\gamma}$; otherwise, $a_{p q}^{i i+1}=0$.

It is obvious that the matrix $M(\gamma)$ is contained in $\Omega_{n}$ and $M(W(A))=A$ for any matrix $A \in \Omega_{n}$.

Example 2.1. (1) Let $A=\left(A_{i j}\right)_{1 \leq i, j \leq 3}$ be the $11 \times 11$ matrix in $\Omega_{3}$ given by

$$
A=\left(\begin{array}{ccccccccccc}
-1 & 0 & 1 & 0 & 0 & 0 & 0 & & & & \\
-1 & 1 & 0 & 0 & 0 & 0 & 0 & & & & \\
0 & 1 & -1 & 0 & 0 & 0 & 0 & & & & \\
1 & 0 & 0 & -1 & 0 & 0 & 1 & 0 & 0 & 0 & 1 \\
0 & -1 & 0 & 1 & -1 & 0 & 0 & 0 & 0 & 0 & 1 \\
0 & 0 & 0 & 0 & -1 & 1 & 0 & 0 & 0 & 0 & 0 \\
0 & 0 & 1 & 0 & 0 & 1 & -1 & 0 & 0 & 0 & 0 \\
& & & 0 & 0 & 0 & 0 & -1 & 0 & 0 & 1 \\
& & & 0 & 0 & -1 & 0 & 1 & -1 & 0 & 0 \\
& & & 0 & 0 & 0 & 0 & 0 & -1 & 1 & 0 \\
& & & 0 & 0 & 0 & 1 & 0 & 0 & -1 & 1
\end{array}\right) .
$$

Then $W_{3}=\sigma_{3} \sigma_{3} \sigma_{3}^{-1} \sigma_{3}^{-1}, W_{2}=\sigma_{2} \sigma_{2} \sigma_{3} \sigma_{3} \sigma_{2}^{-1} \sigma_{3}^{-1} \sigma_{3}^{-1} \sigma_{2}$, and

$$
W_{1}=\sigma_{2} \sigma_{1} \sigma_{2} \sigma_{1}^{-1} \sigma_{3} \sigma_{3} \sigma_{2}^{-1} \sigma_{3}^{-1} \sigma_{3}^{-1} \sigma_{2} \sigma_{1}=W(A) \in \mathcal{F}_{3} .
$$

Conversely, let $\gamma=W(A)$. Then the rewritten word $\bar{\gamma}$ of $\gamma$ is

$$
\bar{\gamma}=\sigma_{(2,1)} \sigma_{(1,1)} \sigma_{(2,2)} \sigma_{(1,2)}^{-1} \sigma_{(3,1)} \sigma_{(3,2)} \sigma_{(2,3)}^{-1} \sigma_{(3,3)}^{-1} \sigma_{(3,4)}^{-1} \sigma_{(2,4)} \sigma_{(1,3)} .
$$

This gives that $M(\gamma)=M(W(A))=A$. 
Now let $\omega=\sigma_{2} \sigma_{1} \sigma_{2} \sigma_{3} \sigma_{3} \sigma_{1}^{-1} \sigma_{2}^{-1} \sigma_{3}^{-1} \sigma_{3}^{-1} \sigma_{2} \sigma_{1} \in \mathcal{F}_{3}$. Then the rewriting word $\bar{\omega}$ of $\omega$ is $\bar{\omega}=\sigma_{(2,1)} \sigma_{(1,1)} \sigma_{(2,2)} \sigma_{(3,1)} \sigma_{(3,2)} \sigma_{(1,2)}^{-1} \sigma_{(2,3)}^{-1} \sigma_{(3,3)}^{-1} \sigma_{(3,4)}^{-1} \sigma_{(2,4)} \sigma_{(1,3)}$ and $M(\omega)=A$. So $W(M(\omega))=W(A)=\gamma$.

(2) Let $A$ and $B$ be the $n \times n$ matrices in $\Omega_{1}$ and $\Omega_{n}$, respectively, given by

$$
A=\left(\begin{array}{cccccc}
-1 & 0 & 0 & \cdots & 0 & 1 \\
1 & -1 & 0 & \cdots & 0 & 0 \\
0 & 1 & -1 & \cdots & 0 & 0 \\
\vdots & \vdots & \vdots & \ddots & \vdots & \vdots \\
0 & 0 & 0 & \cdots & -1 & 0 \\
0 & 0 & 0 & \cdots & 1 & -1
\end{array}\right), B=\left(\begin{array}{cccccc}
-1 & 1[0] & 0 & \cdots & 0 & 0 \\
0[1] & -1 & 1[0] & \cdots & 0 & 0 \\
0 & 0[1] & -1 & \cdots & 0 & 0 \\
\vdots & \vdots & \vdots & \ddots & \vdots & \vdots \\
0 & 0 & 0 & \cdots & -1 & 1[0] \\
0 & 0 & 0 & \cdots & 0[1] & -1
\end{array}\right)
$$

Then $W(A)=\sigma_{1}^{n} \in \mathcal{F}_{1}$ and $W(B)=\sigma_{1} \sigma_{2} \cdots \sigma_{n}\left[\sigma_{n} \sigma_{n-1} \cdots \sigma_{1}\right]$ in $\mathcal{F}_{n}$. Conversely, $M(W(A))=M\left(\sigma_{1}^{n}\right)=A$ and $M(W(B))=M\left(\sigma_{1} \sigma_{2} \cdots \sigma_{n}\left[\sigma_{n} \sigma_{n-1} \cdots \sigma_{1}\right]\right)=B$.

Let $\gamma$ and $\omega$ be two words in $\mathcal{F}_{n}$. By $\gamma \approx \omega$ we mean that $\gamma$ can be transformed to $\omega$ by applying a finite number of relations: $\sigma_{i}^{ \pm 1} \sigma_{j}^{ \pm 1}=\sigma_{j}^{ \pm 1} \sigma_{i}^{ \pm 1}(|i-j| \geq 2)$ and vice versa. For $\gamma \in \mathcal{F}_{n}$, the equivalence class of $\gamma$ is denoted by $[\gamma]$, i.e., $[\gamma]=\left\{\omega \in \mathcal{F}_{n} \mid \omega \approx \gamma\right\}$.

Lemma 2.2. Let $\gamma$ and $\omega$ be two words in $\mathcal{F}_{n}$. Then $\gamma \approx \omega$ if and only if $M(\gamma)=$ $M(\omega)$.

Proof. Let $M(\gamma)=\left(A_{i j}\right)_{1 \leq i, j \leq n}$ and $M(\omega)=\left(B_{i j}\right)_{1 \leq i, j \leq n}$ be the matrices for $\gamma$ and $\omega$ in $\Omega_{n}$ defined as above. For each $i \in\{1,2, \cdots, n\}$, let $\gamma(i)$ and $\omega(i)$ denote the words obtained from $\gamma$ and $\omega$ by replacing all generators $\sigma_{j}$ and $\sigma_{j}^{-1}(j \neq i)$ with the empty word and similarly, for $i \in\{1,2, \cdots, n-1\}$, let $\gamma(i, i+1)$ and $\omega(i, i+1)$ denote the words obtained from $\gamma$ and $\omega$ by replacing all generators $\sigma_{j}$ and $\sigma_{j}^{-1}(j \neq i, i+1)$ with the empty word, respectively. Then it is clear from the definition that the words $\gamma(i), \gamma(i+1)$ and $\gamma(i, i+1)$ uniquely define the blocks $A_{i i}, A_{i+1 i+1}, A_{i i+1}$, and $A_{i+1 i}$ of $M(\gamma)$ and the words $\omega(i), \omega(i+1)$ and $\omega(i, i+1)$ uniquely define the blocks $B_{i i}, B_{i+1 i+1}, B_{i i+1}$, and $B_{i+1 i}$ of $M(\omega)$, and vice versa. Now $\gamma \approx \omega$ if and only if $\gamma(i)=\omega(i)(1 \leq i \leq n)$ and $\gamma(i, i+1)=\omega(i, i+1)(1 \leq i \leq n-1)$, equivalently $A_{i i}=B_{i i}(1 \leq i \leq n)$ and $A_{i+1 i}=B_{i+1 i}, A_{i i+1}=B_{i i+1}(1 \leq i \leq n-1)$. Hence $\gamma \approx \omega$ if and only if $M(\gamma)=M(\omega)$.

Lemma 2.3. For any word $\gamma$ in $\mathcal{F}_{n}, W(M(\gamma)) \approx \gamma$.

Proof. Let $\gamma$ be any word in $\mathcal{F}_{n}$ and let $M(\gamma)$ be the matrix for $\gamma$ in $\Omega_{n}$. Now let $\omega=W(M(\gamma))$. Then $M(\omega)=M(W(M(\gamma)))=M(\gamma)$. By Lemma 2.2, $\gamma \approx \omega=$ $W(M(\gamma))$.

Theorem 2.4. Let $\Omega_{n}$ be the set of all block tridiagonal matrices described in (2.1) and let $\left[\mathcal{F}_{n}\right]=\left\{[\gamma] \mid \gamma \in \mathcal{F}_{n}\right\}$, where $[\gamma]=\left\{\omega \in \mathcal{F}_{n} \mid \omega \approx \gamma\right\}$. Then $\Omega_{n}$ and $\left[\mathcal{F}_{n}\right]$ are in one-to-one correspondence.

Proof. Let $\Phi:\left[\mathcal{F}_{n}\right] \rightarrow \Omega_{n}$ be the mapping defined by $\Phi([\gamma])=M(\gamma)$ for $[\gamma] \in\left[\mathcal{F}_{n}\right]$ and let $\Psi: \Omega_{n} \rightarrow\left[\mathcal{F}_{n}\right]$ be the mapping defined by $\Psi(A)=[W(A)]$ for $A \in \Omega_{n}$. By Lemma 2.2, $\Phi$ is well defined and, by Lemma 2.3, $\Psi(\Phi([\gamma]))=[W(M(\gamma))]=[\gamma]$ for any $[\gamma] \in\left[\mathcal{F}_{n}\right]$. Since $\Phi(\Psi(A))=M(W(A))=A$ for any matrix $A \in \Omega_{n}$, $\Phi$ is a bijection and $\Phi^{-1}=\Psi$. This completes the proof. 
Let $B_{n+1}$ be the Artin's braid group on $n+1$ strings and let $\gamma$ be any braid word of $B_{n+1}$. Then we may regard $\gamma$ as an element of $\mathcal{F}_{n}$. In this regard, the trivial braid $1_{n+1}$ of $B_{n+1}$ corresponds to the empty word in $\mathcal{F}_{n}$. This gives that each braid word $\gamma \in B_{n+1}$ can be presented by a matrix $M(\gamma) \in \Omega_{n}$ and, conversely, any matrix $A \in \Omega_{n}$ presents a braid word $W(A) \in B_{n+1}$. In what follows the matrix $M(\gamma) \in \Omega_{n}$ corresponding to the braid word $\gamma \in B_{n+1}$ is called the matrix presentation of the braid word $\gamma$. Note that each braid of $B_{n+1}$ has many matrix presentations.

\section{Alexander matrices for Closed BRAids}

Let $A=\left(A_{i j}\right)_{1 \leq i, j \leq n}$ be a matrix in $\Omega_{n}$, where $A_{i i}$ is a $s_{i} \times s_{i}$ matrix $\left(s_{i} \geq 1\right)$, and let

$$
\bar{A}=\left(\begin{array}{cccccc}
\bar{A}_{11} & & & & & \\
\bar{A}_{21} & \bar{A}_{22} & & & & \\
& \bar{A}_{32} & \bar{A}_{33} & & & \\
& & \ddots & \ddots & & \\
& & & \bar{A}_{n-1 n-2} & \bar{A}_{n-1 n-1} & \bar{A}_{n n}
\end{array}\right),
$$

where $\bar{A}_{i i}=A_{i i}(1 \leq i \leq n)$ and $\bar{A}_{i+1 i}=A_{i+1 i}+A_{i i+1}^{T}(1 \leq i \leq n-1)$.

Define $D_{A}=\bar{A}_{11} \oplus \cdots \oplus \bar{A}_{n n}$ and $L_{A}=\bar{A}-D_{A}$.

Throughout this section we denote by $C$ and $E$ the block diagonal matrices given by $C=C_{s_{1}} \oplus \cdots \oplus C_{s_{n}}$ and $E=E_{s_{1}} \oplus \cdots \oplus E_{s_{n}}$, where $C_{s_{i}}$ is a $s_{i} \times s_{i}$ circulant matrix and $E_{s_{i}}$ is a $s_{i} \times s_{i}$ skew-symmetric matrix: $C_{s_{i}}=E_{s_{i}}=(0)$ if $s_{i}=1, E_{s_{i}}=O_{2 \times 2}$ if $s_{i}=2$, and

$$
C_{s_{i}}=\left(\begin{array}{cccccc}
1 & -1 & 0 & \cdots & 0 & 0 \\
0 & 1 & -1 & \cdots & 0 & 0 \\
0 & 0 & 1 & \cdots & 0 & 0 \\
\vdots & \vdots & \vdots & \ddots & \vdots & \vdots \\
0 & 0 & 0 & \cdots & 1 & -1 \\
-1 & 0 & 0 & \cdots & 0 & 1
\end{array}\right), E_{s_{i}}=\frac{1}{2}\left(\begin{array}{cccccc}
0 & 1 & 0 & \cdots & 0 & -1 \\
-1 & 0 & 1 & \cdots & 0 & 0 \\
0 & -1 & 0 & \cdots & 0 & 0 \\
\vdots & \vdots & \vdots & \ddots & \vdots & \vdots \\
0 & 0 & 0 & \cdots & 0 & 1 \\
1 & 0 & 0 & \cdots & -1 & 0
\end{array}\right)
$$

Let $Z$ be an infinite cyclic group generated by $t$ and let $J Z$ be the integral group ring of $Z$. For a matrix $A \in \Omega_{n}$, let $\Lambda_{A}(t)$ be the matrix with entries in the ring $J Z$ defined by

$$
\Lambda_{A}(t)=L_{A} D_{A}-t\left(L_{A} D_{A}\right)^{T}+\frac{1}{2}(1-t) C D_{A}+(1+t) E .
$$

Then we have the following

Theorem 3.1. Let $\gamma$ be a braid in $B_{n+1}$ and let $M(\gamma) \in \Omega_{n}$ be a matrix presentation of $\gamma$. Then a reduced Alexander matrix for the closure $\gamma^{\wedge}$ of $\gamma$ is given by

$$
\Lambda_{M(\gamma)}(t)=L_{M(\gamma)} D_{M(\gamma)}-t\left(L_{M(\gamma)} D_{M(\gamma)}\right)^{T}+\frac{1}{2}(1-t) C D_{M(\gamma)}+(1+t) E .
$$

Proof. The theorem follows from Theorem 4.1([Mur]) and the identity $M^{\prime}(\gamma)-$ $t M^{\prime}(\gamma)^{T}=\Lambda_{M(\gamma)}(t)$, where $M^{\prime}(\gamma)$ is the Murasugi's matrix with respect to $\gamma^{\wedge}$ given by the following: 


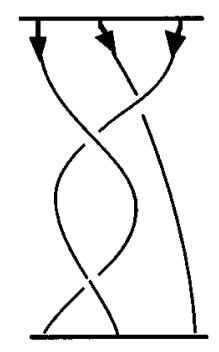

$\gamma$

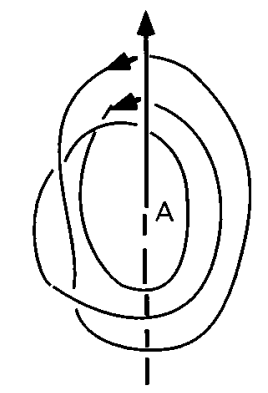

$\gamma^{\wedge}=$ the closure of $\gamma$

FigURE 3.1

Lemma 3.2. Let $\gamma$ be a braid word in $B_{n+1}$ and let $A=M(\gamma) \in \Omega_{n}$ be the matrix presentation of $\gamma$. Then $M^{\prime}(\gamma)=\left(L_{A}+\frac{1}{2} C\right) D_{A}+E$ is a Murasugi's matrix with respect to the closure $\gamma^{\wedge}$ of $\gamma$. Consequently, if two braids $\gamma$ and $\omega$ are Markov equivalent, then $M^{\prime}(\gamma)$ and $M^{\prime}(\omega)$ are $S$-equivalent.

Proof. Let $\gamma$ be a braid word in $B_{n+1}$ and let $M(\gamma)=\left(A_{i j}\right)_{1 \leq i, j \leq n}$ be the matrix presentation in $\Omega_{n}$ of $\gamma$. Assume that each $A_{i i}$ in $M(\gamma)$ is an $s_{i} \times s_{i}$ matrix $\left(s_{i} \geq 1\right)$. The closure $\gamma^{\wedge}$ of $\gamma$ is an oriented link diagram in $\mathbb{R}^{2}$ obtained from $\gamma$ by joining the $n+1$ points at the top of the braid $\gamma$ to the corresponding $n+1$ points at the bottom without further crossings as in Figure 3.1.

In what follows we refer to [Mur, pp. 389-391] for the definitions of the Seifert circuit, Seifert domain, $\alpha$-region, $\beta$-region, and the indices $\eta(c), d_{r}(c)$ and $\epsilon_{r}(c)$.

Case I. $\gamma$ involves all the generators $\sigma_{1}, \sigma_{2}, \cdots, \sigma_{n}$ of $B_{n+1}$. It is obvious that the number of Seifert circuits in $\gamma^{\wedge}$ is equal to $n+1$. Among them, $n-1$ Seifert circuits are of the second type and so there are $n$ Seifert domains, say, $D_{1}, D_{2}, \cdots, D_{n}$. Note that the unbounded region in $D_{1}$ and the region in $D_{n}$ which intersects the braid axis $A$ of $\gamma^{\wedge}$ are $\beta$-regions. Otherwise, they are all $\alpha$-regions.

Now let $\bar{\gamma}$ be the rewriting word of $\gamma \in B_{n+1}$ and, for each $i \in\{1,2, \cdots, n\}$, let $W_{p}^{i}\left(p=1,2, \cdots, s_{i}\right)$ denote the word obtained from $\bar{\gamma}$ such that the initial letter of $W_{p}^{i}$ is $\sigma_{(i, p)}^{\tau(i, p)}$ and the terminal letter of $W_{p}^{i}$ is $\sigma_{(i, p+1)}^{\tau(i, p+1)}\left(s_{i}+1=1\right)$ cyclically. Define $\bar{W}_{p}^{i}\left(p=1,2, \cdots, s_{i}\right)$ to be the word obtained from $W_{p}^{i}$ by replacing all $\sigma_{(k, q)}^{\tau(k, q)}(k \neq i-1, i, i+1)$ with the empty word. Then each $D_{i}(i=1,2, \cdots, n)$ contains $s_{i} \alpha$-regions, denoted by $X_{1}^{i}, X_{2}^{i}, \cdots, X_{s_{i}}^{i}$, which can be identified with the words $\bar{W}_{1}^{i}, \bar{W}_{2}^{i}, \cdots, \bar{W}_{s_{i}}^{i}$, respectively, in such a way that all vertices in the boundary of the region $X_{p}^{i}$ are just the letters in $\bar{W}_{p}^{i}\left(p=1,2, \cdots, s_{i}\right)$.

From Definition 3.3 [Mur, p.391] and the identification of $X_{p}^{i}$ with $\bar{W}_{p}^{i}$, Murasugi's matrix $M$ with respect to $\gamma^{\wedge}$ is given by the block matrix:

$$
\begin{gathered}
M=\left(M_{i j}\right)_{i, j=1,2, \cdots, n}, \\
M_{i i}=\left(a_{p q}^{(i)}\right)_{p, q=1,2, \cdots, s_{i}},
\end{gathered}
$$

where for $p \neq q a_{p q}^{(i)}=-\sum \eta(c) d_{\bar{W}_{p}^{i}}(c)$, where the summation extends over all common letters $c$ that are in the two different $\alpha$-regions $\bar{W}_{p}^{i}$ and $\bar{W}_{q}^{i}$ contained in 
$D_{i}$, and $a_{p p}^{(i)}=-\sum_{q=1, p \neq q}^{s_{i}} a_{p q}^{(i)}$,

$$
M_{i j}=\left(b_{p q}^{(i j)}\right)_{p=1,2, \cdots, s_{i} ; q=1,2, \cdots, s_{j}}(i \neq j),
$$

where $b_{p q}^{(i j)}=-\sum \eta(c) d_{\bar{W}_{p}^{i}}(c) \epsilon_{\bar{W}_{q}^{j}}(c)$, where the summation extends over all common letters $c$ that are in the two $\alpha$-regions $\bar{W}_{p}^{i}$ and $\bar{W}_{q}^{j}$ contained in $D_{i}$ and $D_{j}$ respectively.

(3.2.1). $M_{i i}=\frac{1}{2} C_{s_{i}} \bar{A}_{i i}+E_{s_{i}}(i=1,2, \cdots, n)$.

If $s_{i}=1$, then $D_{i}$ has one and only one $\alpha$-region $\bar{W}_{1}^{i}$, and $M_{i i}=(0)$.

If $s_{i}=2$, then

$$
M_{i i}=\left(a_{p q}^{(i)}\right)_{p, q=1,2}=\left(\begin{array}{cc}
-\frac{\tau(i, 1)+\tau(i, 2)}{2} & \frac{\tau(i, 1)+\tau(i, 2)}{2} \\
\frac{\tau(i, 1)+\tau(i, 2)}{2} & -\frac{\tau(i, 1)+\tau(i, 2)}{2}
\end{array}\right)=\frac{1}{2} C_{s_{i}} \bar{A}_{i i} .
$$

If $s_{i} \geq 3$, then for $1 \leq p, q \leq s_{i}(p \neq q)$,

$$
a_{p q}^{(i)}= \begin{cases}\frac{\tau(i, p)-1}{2} & \text { if } p=q+1\left(1 \leq q \leq s_{i}-1\right) \text { or } p=1, q=s_{i}, \\ \frac{1+\tau(i, q)}{2} & \text { if } q=p+1\left(1 \leq p \leq s_{i}-1\right) \text { or } p=s_{i}, q=1, \\ 0 & \text { otherwise. }\end{cases}
$$

So $M_{i i}=\left(a_{p q}^{(i)}\right)_{p, q=1,2, \cdots, s_{i}}$ is the matrix given by $M_{i i}=$

$$
\begin{aligned}
& \left(\begin{array}{cccccc}
-\frac{\tau(i, 1)+\tau(i, 2)}{2} & \frac{1+\tau(i, 2)}{2} & 0 & \cdots & 0 & \frac{\tau(i, 1)-1}{2} \\
\frac{\tau(i, 2)-1}{2} & -\frac{\tau(i, 2)+\tau(i, 3)}{2} & \frac{1+\tau(i, 3)}{2} & \cdots & 0 & 0 \\
0 & \frac{\tau(i, 3)-1}{2} & -\frac{\tau(i, 3)+\tau(i, 4)}{2} & \cdots & 0 & 0 \\
\vdots & \vdots & \vdots & \ddots & \vdots & \vdots \\
0 & 0 & 0 & \cdots & -\frac{\tau\left(i, s_{i}-1\right)+\tau\left(i, s_{i}\right)}{2} & \frac{1+\tau\left(i, s_{i}\right)}{2} \\
\frac{1+\tau(i, 1)}{2} & 0 & 0 & \cdots & \frac{\tau\left(i, s_{i}\right)-1}{2} & -\frac{\tau(i, 1)+\tau\left(i, s_{i}\right)}{2}
\end{array}\right) \\
& =\frac{1}{2} C_{s_{i}} \bar{A}_{i i}+E_{s_{i}} .
\end{aligned}
$$

(3.2.2). For $1 \leq i, j \leq n(i \neq j), M_{i j}= \begin{cases}\bar{A}_{i j} \bar{A}_{j j} & \text { if } i=j+1, \\ O_{s_{i} \times s_{j}} & \text { otherwise. }\end{cases}$

(i) $|i-j|>2$ : there do not exist common letters that are in the two $\alpha$-regions $\bar{W}_{p}^{i}$ and $\bar{W}_{q}^{j}$ for all $1 \leq p \leq s_{i}, 1 \leq q \leq s_{j}$. Hence $b_{p q}^{(i j)}=0$ and so $M_{i j}=O_{s_{i} \times s_{j}}$.

(ii) $|i-j|=1: b_{p q}^{(i+1 i)}=-\sum \eta(c) d_{\bar{W}_{p}^{i+1}}(c) \epsilon_{\bar{W}_{q}^{i}}(c)$, where the summation extends over all letters that are in the two $\alpha$-regions $\bar{W}_{p}^{i+1}$ and $\bar{W}_{q}^{i}$. Since $d_{\bar{W}_{p}^{i+1}}(c)=0$ for all common letters $c$ that are not in the two regions $\bar{W}_{p}^{i+1}$ and $\bar{W}_{q}^{i}$, we have

$$
\begin{aligned}
b_{p q}^{(i+1 i)} & =-\sum_{k=1}^{s_{i}} \eta\left(\sigma_{(i, k)}^{\tau(i, k)}\right) d_{\bar{W}_{p}^{i+1}}\left(\sigma_{(i, k)}^{\tau(i, k)}\right) \epsilon_{\bar{W}_{q}^{i}}\left(\sigma_{(i, k)}^{\tau(i, k)}\right) \\
& -\sum_{k=1}^{s_{i+1}} \eta\left(\sigma_{(i+1, k)}^{\tau(i+1, k)}\right) d_{\bar{W}_{p}^{i+1}}\left(\sigma_{(i+1, k)}^{\tau(i+1, k)}\right) \epsilon_{\bar{W}_{q}^{i}}\left(\sigma_{(i+1, k)}^{\tau(i+1, k)}\right) .
\end{aligned}
$$

Since each letter $\sigma_{(i+1, k)}^{\tau(i+1, k)}$ does not belong to $D_{i}$ (for the definition, see [Mur, p.390]), $\epsilon_{\bar{W}_{q}^{i}}\left(\sigma_{(i+1, k)}^{\tau(i+1, k)}\right)=0$ for all $k=1,2, \cdots, s_{i+1}$. 
Now let $\bar{a}_{p k}^{i+1 i}=-\eta\left(\sigma_{(i, k)}^{\tau(i, k)}\right) d_{\bar{W}_{p}^{i+1}}\left(\sigma_{(i, k)}^{\tau(i, k)}\right)$. Then

$$
\bar{a}_{p k}^{i+1 i}= \begin{cases}0 & \text { if } \sigma_{(i, k)}^{\tau(i, k)} \text { is not in } \bar{W}_{p}^{i+1}, \\ \tau(i, k) & \text { if } \sigma_{(i, k)}^{\tau(i, k)} \text { is in } \bar{W}_{p}^{i+1} .\end{cases}
$$

This gives that $\left(\bar{a}_{p k}^{i+1 i}\right)_{1 \leq p \leq s_{i+1}, 1 \leq k \leq s_{i}}=\bar{A}_{i+1 i}$. Let $\bar{a}_{k q}^{i i}=\epsilon_{\bar{W}_{q}^{i}}\left(\sigma_{(i, k)}^{\tau(i, k)}\right)$. Then we can check that $\left(\bar{a}_{k q}^{i i}\right)_{1 \leq k, q \leq s_{i}}=\bar{A}_{i i}$. Hence

$$
\begin{aligned}
M_{i+1 i} & =\left(b_{p q}^{(i+1 i)}\right)_{p=1,2, \cdots, s_{i+1} ; q=1,2, \cdots, s_{i}} \\
& =\left(-\sum_{k=1}^{s_{i}} \eta\left(\sigma_{(i, k)}^{\tau(i, k)}\right) d_{\bar{W}_{p}^{i+1}}\left(\sigma_{(i, k)}^{\tau(i, k)}\right) \epsilon_{\bar{W}_{q}^{i}}\left(\sigma_{(i, k)}^{\tau(i, k)}\right)\right)_{p=1,2, \cdots, s_{i+1} ; q=1,2, \cdots, s_{i}} \\
& =\left(\sum_{k=1}^{s_{i}} \bar{a}_{p k}^{i+1 i} \bar{a}_{k q}^{i i}\right)_{p=1,2, \cdots, s_{i+1} ; q=1,2, \cdots, s_{i}} \\
& =\bar{A}_{i+1 i} \bar{A}_{i i} .
\end{aligned}
$$

It is obvious that $M_{i i+1}=O_{s_{i} \times s_{i+1}}$.

(iii) $|i-j|=2$ : Since each letter $c$ that is in the two $\alpha$-regions $X_{p}^{i}$ and $X_{q}^{j}$ for all $p, q$ are not contained in $D_{i}$ or $D_{j}, \epsilon_{\bar{W}_{q}^{j}}(c)=0$. Hence

$$
M_{i j}=\left(b_{p q}^{(i j)}\right)_{p=1,2, \cdots, s_{i} ; q=1,2, \cdots, s_{j}}=\left(-\sum \eta(c) d_{\bar{W}_{p}^{i}}(c) \epsilon_{\bar{W}_{q}^{j}}(c)\right)=O_{s_{i} \times s_{j}} .
$$

Combining (3.2.1) and (3.2.2), we obtain that $M=L_{A} D_{A}+\frac{1}{2} C D_{A}+E=M^{\prime}(\gamma)$.

Case II. $\gamma$ does not involve the generator $\sigma_{i_{1}}, \cdots, \sigma_{i_{k}}\left(i_{j} \in\{1,2, \cdots, n\}\right)$ with $i_{1}<i_{2}<\cdots<i_{k}$. Then $\gamma \approx \omega=\omega_{1}\left(1, i_{1}-1\right) \omega_{2}\left(i_{1}+1, i_{2}-1\right) \cdots \omega_{k}\left(i_{k}+1, n\right)$, where for $1 \leq j \leq k, \omega_{j}(r, s)$ denotes a braid word which involves all generators $\sigma_{r}, \sigma_{r+1}, \cdots, \sigma_{s}$ if $r \leq s$, and $\omega_{j}(r, s)$ denotes the empty word if $r>s$. By Lemma $2.2, M(\gamma)=M(\omega)$ and so $M^{\prime}(\gamma)=M^{\prime}(\omega)=M^{\prime}\left(\omega_{1}\right) \oplus(0) \oplus M^{\prime}\left(\omega_{2}\right) \oplus(0) \oplus \cdots \oplus$ (0) $\oplus M^{\prime}\left(\omega_{k}\right)$, where $M^{\prime}\left(\omega_{j}\right)=M^{\prime}\left(\omega_{j}(r, s)\right)$ is the empty matrix if $r>s$. Note that the closure $\omega^{\wedge}$ is a split diagram with $k$ components $\omega_{1}^{\wedge}, \omega_{2}^{\wedge}, \cdots, \omega_{k}^{\wedge}$. Let $M_{j}$ denote the Murasugi's matrix with respect to $\omega_{j}^{\wedge}$. Then by Case I, we see that $M_{j}=M^{\prime}\left(\omega_{j}\right)$. Hence the Murasugi's matrix $M$ with respect to $\omega^{\wedge}$ is the matrix $M=M_{1} \oplus \cdots \oplus M_{k} \oplus O_{(k-1) \times(k-1)}$ and there is a unimodular integral matrix $U$ such that $U M U^{T}=M^{\prime}(\omega)$. This completes the proof of Lemma 3.2 and so Theorem 3.1 .

Now let $\Lambda_{M(\gamma)}^{*}(t)$ denote the principal minor of the reduced Alexander matrix $\Lambda_{M(\gamma)}(t)$ for $\gamma^{\wedge}$ obtained by deleting the row and column containing $(1,1)$-entry in each diagonal block matrix $\frac{1}{2}(1-t) C_{s_{i}} \bar{A}_{i i}+(1+t) E_{s_{i}}$ corresponding to the nonzero block $\bar{A}_{i i}$ of $D_{A}$. Then we have the following:

Theorem 3.3. Let $\gamma$ be a braid word in $B_{n+1}$ and let $\gamma^{\wedge}$ be the closure of $\gamma$. Then $\sigma\left(\gamma^{\wedge}\right)=\sigma\left(\Lambda_{M(\gamma)}^{*}(-1)\right)$ and $\mathcal{N}\left(\gamma^{\wedge}\right)=\mathcal{N}\left(\Lambda_{M(\gamma)}^{*}(-1)\right)+1$

Proof. Let $M^{\prime}(\gamma)$ be the Murasugi matrix with respect to $\gamma^{\wedge}$ given by Lemma 3.2 and let $M^{*}(\gamma)$ denote the principal minor of $M^{\prime}(\gamma)$ obtained by deleting the row and column containing $(1,1)$-entry in each diagonal block matrix $\frac{1}{2} C_{s_{i}} \bar{A}_{i i}+E_{s_{i}}$ corresponding to the nonzero block $\bar{A}_{i i}$ of $D_{A}$. Then $\sigma\left(\gamma^{\wedge}\right)=\sigma\left(M^{*}(\gamma)+M^{*}(\gamma)^{T}\right)$ and $\mathcal{N}\left(\gamma^{\wedge}\right)=\mathcal{N}\left(M^{*}(\gamma)+M^{*}(\gamma)^{T}\right)+1$. But $M^{*}(\gamma)+M^{*}(\gamma)^{T}=\Lambda_{M(\gamma)}^{*}(-1)$. This completes the proof. 
Theorem 3.4. Let $A$ be any matrix in $\Omega_{n}$ and let $W(A)$ be the braid in $B_{n+1}$ corresponding to $A$. Then $\sigma\left(W(A)^{\wedge}\right)=\sigma\left(\Lambda_{A}^{*}(-1)\right)$ and $\mathcal{N}\left(W(A)^{\wedge}\right)=\mathcal{N}\left(\Lambda_{A}^{*}(-1)\right)+1$.

Proof. Since $M(W(A))=A, \Lambda_{M(W(A))}^{*}(t)=\Lambda_{A}^{*}(t)$. By Theorem 3.3, $\sigma\left(W(A)^{\wedge}\right)$ $=\sigma\left(\Lambda_{M(W(A))}^{*}(-1)\right)=\sigma\left(\Lambda_{A}^{*}(-1)\right)$ and $\mathcal{N}\left(W(A)^{\wedge}\right)=\mathcal{N}\left(\Lambda_{M(W(A))}^{*}(-1)\right)+1=$ $\mathcal{N}\left(\Lambda_{A}^{*}(-1)\right)+1$.

Remark 3.5. For $i \in\{1,2, \cdots, n\}$, let $B_{n+1}^{i}$ denote the subgroup of $B_{n+1}$ generated by $\sigma_{1}, \cdots, \sigma_{i-1}, \sigma_{i+1}, \cdots, \sigma_{n}$. A braid $\gamma \in B_{n+1}$ is called a split braid if $\gamma$ is conjugate into $B_{n+1}^{i}$ for some $i \in\{1,2, \cdots, n\}$. A braid $\gamma \in B_{n+1}$ is called a positive braid if it can be presented by a braid word such that the exponents of the generators in the word are all positive.

(1) Let $\gamma$ be a nonsplit braid word in $B_{n+1}$. Then $\gamma$ involves all generators in $B_{n+1}$ and so $\ell(\gamma)=\mathcal{R}\left(\Lambda_{M(\gamma)}^{*}(-1)\right)+\mathcal{N}\left(\gamma^{\wedge}\right)+n-1$, where $\ell(\gamma)$ is the letter length of $\gamma$ and $\mathcal{R}\left(\Lambda_{M(\gamma)}^{*}(-1)\right)$ denotes the rank of the matrix $\Lambda_{M(\gamma)}^{*}(-1)$. Hence we can see that $\left|\sigma\left(\gamma^{\wedge}\right)\right| \leq \mathcal{L}(\gamma)-\mathcal{N}\left(\gamma^{\wedge}\right)-n+1$, where $\mathcal{L}(\gamma)=\min \{\ell(\omega) \mid \omega$ is equivalent to $\gamma\}$. This shows that if $\ell(\gamma)=\left|\sigma\left(\gamma^{\wedge}\right)\right|+\mathcal{N}\left(\gamma^{\wedge}\right)+n-1$, then $\gamma$ is a minimal length braid representation in $B_{n+1}$ (cf. [Ke]).

(2) Let $\gamma$ be a nonsplit positive braid in $B_{n+1}$ and let $\Delta_{\gamma^{\wedge}}(t)$ denote the reduced Alexander polynomial of $\gamma^{\wedge}$. Since $\operatorname{deg}\left(\Delta_{\gamma^{\wedge}}(t)\right)=\ell(\gamma)-n, \sigma\left(\gamma^{\wedge}\right)+\mathcal{N}\left(\gamma^{\wedge}\right)-1 \leq$ $\operatorname{deg}\left(\Delta_{\gamma^{\wedge}}(t)\right)$. Moreover, the equality holds if and only if $\Lambda_{M(\gamma)}^{*}(-1)$ is a positive semidefinite, that is, the eigenvalues of $\Lambda_{M(\gamma)}^{*}(-1)$ are all nonnegative.

\section{ACKNOWLEDGEMENTS}

The author would like to thank Professors Jin Ho Kwak, Kunio Murasugi, and Chan-Young Park for their valuable comments. He is also grateful to the referee for some corrections and suggestions.

\section{REFERENCES}

[Al] J. W. Alexander, A lemma on systems of knotted curves., Proc. Nat. Acad. Sci. USA 9 (1923), 93-95.

[Bir] J.S. Birman, Braids, links, and mapping class groups, Ann. of Math. Studies, no.82, Princeton Univ. Press, 1975. MR 51:11477

[GL] C. McA. Gordon and R. A. Litherland, On the signature of a link, Invent. Math. 47 (1978), 53-69. MR 58:18407

[Ke] R. D. Keever, Minimal 3-braid representations, J. of Knot Theory and its Ramifications 3 (1994), 163-177. MR 95c:47012

[Mur] K. Murasugi, On a certain numerical invariant of link types, Trans. Amer. Math. Soc. 117 (1965), 387-422. MR 30:1506

[Tr] L. Traldi, On the Goeritz Matrix of a link, Math. Z. 188 (1985), 203-213. MR 87b:57010

Department of Mathematics, College of Natural Science, Kyungpook National UniVERSITY, TAEGU 702-701, KOREA

E-mail address: syleek@chollian.dacom.co.kr

Current address: Department of Mathematics, Pusan National University, Pusan 609-735, Korea

E-mail address: syleek@chollian.net 